\title{
Predicting gastrointestinal and renal involvement in adult IgA vasculitis
}

\author{
Alojzija Hočevar ${ }^{1,2^{*}}$, Matija Tomšič ${ }^{1,2}$, Vesna Jurčić ${ }^{3}, K^{2}$ atja Perdan Pirkmajer ${ }^{1}$ and Žiga Rotar $^{1}$
}

\begin{abstract}
Background: Immunoglobulin A vasculitis (IgAV) is still poorly defined in the adult population. We aimed to determine the predictors of gastrointestinal $(G I)$ or renal involvement in adult IgAV.

Methods: The prospective study included histologically proven adult IgAV cases diagnosed between January 2013 and July 2019 at our secondary/tertiary rheumatology center. We evaluated the role of clinical and the laboratory parameters as markers predicting the Gl or renal involvement in IgAV, using the multiple logistic regression analysis.

Results: During the 79-month observation period, we identified 214 new adult IgAV cases (59.3\% males, median (interquartile range) age 64.6 (57.2-76.7) years). The Gl tract and renal involvement developed in 58 (27.1\%) and 83 (38.8\%) cases, respectively (concurrently in $26(12.1 \%)$ cases). In the multivariate logistic regression analysis, generalized purpura (OR $6.74(95 \% \mathrm{Cl} 3.18-14.31))$, the pre-treatment neutrophil to lymphocyte ratio (NLR) > 3.5 (OR $2.78(95 \% \mathrm{Cl}$ 1.34-5.75)), and elevated serum IgA levels (OR $0.40(95 \% \mathrm{Cl} 0.20-0.79))$ were extracted as factors associated with $\mathrm{Gl}$ complications, whereas current smoking (OR 3.23 (95\%Cl 1.50-6.98)), generalized purpura (OR 1.98 (95\%Cl 1.08-3.61)), elevated serum IgA (OR 2.25 (95\%Cl 1.21-4.18)), NLR > 3.5 (OR 1.96 (95\%Cl 1.02-3.77)), and marginally age (1.02 (95\%Cl 1.01-1.04)) emerged as factors associated with renal complications.
\end{abstract}

Conclusion: Generalized purpura and pre-treatment NLR predicted both Gl and renal involvement, whereas active smoking was associated with renal involvement, and the serum IgA level had a divergent effect on renal and Gl involvement in adult IgAV.

Keywords: IgA vasculitis, Gastrointestinal involvement, Renal involvement

\section{Introduction}

Immunoglobulin A vasculitis (IgA) is an immune complex small vessel leukocytoclastic vasculitis that commonly affects the skin, joints, gastrointestinal (GI) tract, and kidneys [1]. IgAV is a typical childhood vasculitis, commonly with a benign, self-limiting course and complete recovery in the young population. In adults, a severe acute disease with significant GI and renal involvement is common [2, 3]. GI involvement with a severe hemorrhage or bowel perforation represents the major risk of mortality in acute adult IgAV, and renal involvement is associated with an increased risk of progression to chronic kidney failure [2]. Nevertheless, the predictors of visceral involvement in acute adult IgAV have not been extensively studied. Cao

\footnotetext{
* Correspondence: alojzija.hocevar@gmail.com

'Department of Rheumatology, University Medical Centre Ljubljana, Vodnikova cesta 62, 1000 Ljubljana, Slovenia

${ }^{2}$ Faculty of Medicine, University of Ljubljana, Ljubljana, Slovenia

Full list of author information is available at the end of the article
}

et al. reported that the risk of significant renal disease increased with patient age, and the presence of necrotic or bullous skin lesions [4]. Patient age forecasted visceral involvement also in the study by Poterucha et al. Adults under the age of 40 had an increased risk of GI involvement, and those over 40 without eosinophils in a skin biopsy had an increased risk of renal involvement [5]. Contrary to St John et al., who reported a predictive value of skin lesions on the upper and lower extremities for significant long-term renal involvement [6], the distribution of skin lesions above the waist was not found to be a reliable indicator of systemic disease in adult IgAV in the study by Poterucha et al. [5]. Nagy et al. described in a retrospective study a significant association between pretreatment elevated neutrophil to lymphocyte ratio (NLR) and the development of GI or renal manifestations [7]. A similar association between an elevated NLR and severe

(c) The Author(s). 2019 Open Access This article is distributed under the terms of the Creative Commons Attribution 4.0 International License (http://creativecommons.org/licenses/by/4.0/), which permits unrestricted use, distribution, and reproduction in any medium, provided you give appropriate credit to the original author(s) and the source, provide a link to the Creative Commons license, and indicate if changes were made. The Creative Commons Public Domain Dedication waiver (http://creativecommons.org/publicdomain/zero/1.0/) applies to the data made available in this article, unless otherwise stated. 
GI or nephritis has recently been described also in childhood IgAV [8].

The aim of our study was to determine the predictors of gastrointestinal and renal involvement in a prospectively collected cohort of adult IgAV.

\section{Methods}

\section{Setting and patient selection}

This prospective study was conducted at the Department of Rheumatology, University Medical Centre Ljubljana, Slovenia. Patients with suspected vasculitis are regularly referred by their general practitioners or other subspecialists to our early intervention clinic, where they are evaluated on the day of the referral, obviating the need for immunosuppressive treatment before referral.

We included adults (i.e., persons aged $\geq 18$ years), with the symptoms and signs compatible with the definition of IgAV according to the 2012 revised International Chapel Hill Consensus Conference Nomenclature of Vasculitides [1], with histologically proven IgAV, diagnosed for the first time between January 2013 and August 2019. Additionally, all patients fulfilled the classification criteria of the European League Against Rheumatism/Paediatric Rheumatology International Trials Organization/Paediatric Rheumatology European Society (EULAR/PRINTO/PRES) criteria for IgAV [9].

\section{Clinical, laboratory, and histological data}

Patients underwent a detailed clinical evaluation (including a structured history of smoking and potential triggers of IgAV, i.e., infection or the use of new medication within a month prior to an IgAV episode, and cancer history), and an extensive laboratory workup, as described previously [10]. The definitions used for the assessment of the skin, renal, and gastrointestinal involvement are provided in Table 1.

The serum immunoglobulin A (IgA) concentration, pre-treatment white blood cell count (WBC), and NLR were measured. Since concurrent infection could influence the NLR, concurrent infections were recorded and included in the analysis.

Skin or renal biopsies were evaluated using bright field microscopy, and direct immunofluorescence.

\section{Statistical analysis}

The results were expressed as a median and interquartile range (IQR) or mean and standard deviation (SD) for metric, and as percentages for categorical variables. To test the differences between IgAV subgroups, we used the Mann-Whitney test for metric and Fisher's exact test for categorical variables. A receiver operating characteristic (ROC) curve was constructed to evaluate the prognostic utility of NLR and to determine the optimal cutoff value. Potential predictors of IgAV GI and renal
Table 1 Definitions of skin, kidney, and gastrointestinal involvement in IgAV

\begin{tabular}{|c|c|}
\hline \multicolumn{2}{|l|}{ Purpura } \\
\hline Localized & $\begin{array}{l}\text { Vasculitic lesions present only below the } \\
\text { waistline }\end{array}$ \\
\hline Generalized & Vasculitic lesions extending above the waistline \\
\hline \multicolumn{2}{|l|}{ Renal involvement } \\
\hline Hematuria & $\begin{array}{l}>5 \text { red blood cells per high power field or } \\
\text { red blood cells casts in the urinary sediment } \\
\text { or hemoglobinuria } \geq 2+\text { on dipstick }\end{array}$ \\
\hline Macrohematuria & $>1500$ red blood cells $/ \mathrm{mm}^{3}$ of urine \\
\hline Proteinuria & urine protein excretion > 300 mg/day \\
\hline $\begin{array}{l}\text { Severe } \\
\text { involvement }\end{array}$ & $\begin{array}{l}\text { nephrotic or nephritic syndrome with an acute } \\
\text { worsening of the renal function, defined as either } \\
\text { an increase in serum creatinine concentration or } \\
\text { a decrease in the glomerular filtration rate } \\
\text { estimated by the MDRD- } 4>25 \% \text { from the } \\
\text { patient's baseline }\end{array}$ \\
\hline Gl involvement & $\begin{array}{l}\text { new onset of diffuse abdominal pain or } \\
\text { gastrointestinal bleeding }\end{array}$ \\
\hline $\begin{array}{l}\text { Severe } \\
\text { involvement }\end{array}$ & bloody diarrhea, ileus or bowel perforation \\
\hline
\end{tabular}

involvement with a $p$ value $<0.2$ were tested using a multiple logistic regression analysis. The significance threshold selected for the final model was set at 0.05.

\section{Results}

Demographic, epidemiological data, and classification criteria

During the 79-month observation period, we identified 214 new IgAV cases (127 (59.3\%) males). The median (IQR) patient age at diagnosis was 64.6 (47.2-76.7, range 18-96) years. There were $42(19.6 \%)$ current smokers (daily smoking on average (SD) 14 (9) cigarettes) and 55 (25.7\%) past smokers. Sixty-nine (32.2\%) and 52 (24.3\%) patients reported prior infection or the use of new medication within a month of the IgAV episode onset, respectively. In $33(15.4 \%)$ patients, a concurrent infection was recorded. Twenty-seven (12.6\%) patients had a history of cancer. The median (IQR) symptom duration before diagnosis was 7 (5-21) days. One-hundred-and-eighty-five $(86.4 \%)$ patients presented with purpura for the first time, while 29 recalled previous episodes of similar skin lesions without a definitive diagnosis in the past.

All 214 patients fulfilled EULAR/PRINTO/PRES classification criteria for IgAV [9]. In addition to skin involvement, in all 214 patients, the skin biopsy was performed and was consistent with IgAV. Sixty-seven out of 214 (31.3\%) patients had skin limited IgAV (i.e., fulfilling mandatory skin criterion and histological criterion); the remaining 68.7\% fulfilled at least one additional item of EULAR/ PRINTO/PRES classification criteria for IgAV (i.e., articular or GI or renal item). 


\section{$\lg A V$ features}

Skin involvement with histologically proven IgAV was present in all 214 patients. Necrotic and bullous lesions developed in 98 (45.8\%) patients. In 105 (49.1\%) patients, purpura was limited to the lower limbs or up to waist level. Vasculitic lesions above the waistline (i.e., generalized purpura) were observed in 109 (50.9\%) patients. Seventy-two (33.6\%) patients reported arthralgia and 29 (13.6\%) developed arthritis. We recorded GI involvement in $58(27.1 \%)$ patients, and $26(12.1 \%)$ of them had concurrent renal involvement. Forty-seven patients reported abdominal pain, 12 patients experienced bloody diarrhea, and 29 patients occult gastrointestinal bleeding. Six patients developed ileus, and in three patients, a bowel perforation occurred. Overall, 15/58 (25.9\%) patients with GI involvement experienced severe GI involvement identified as severe bloody diarrhea, ileus, or bowel perforation.

Kidney involvement developed in 83 (38.8\%) patients. Fourteen (6.5\%) patients had macrohematuria, and the remaining, microhematuria. Proteinuria developed in 50 (23.4\%) patients. Fifteen patients had daily proteinuria from 1.0 to 3.5 g. Nephrotic range proteinuria was detected in 13 patients. Acute kidney injury developed as a result of vasculitis in 23 (10.7\%) patients. Overall, 30 (14.0\%) patients had a severe course of kidney involvement, defined as the development of acute kidney injury or nephrotic syndrome. A renal biopsy was performed in $9(4.2 \%)$ patients. Proliferative glomerulonephritis was noted in 5 patients, crescentic glomerulonephritis in 3 cases, and predominantly sclerotic glomerular lesions in one patient.

The characteristics of IgAV patients with and without GI or renal involvement are presented in Table 2. The subgroup of patients with renal involvement was significantly older compared to the subgroup with GI involvement (median (IQR) age 67.8 (53.6-77.4) vs. 55.5 (39.0-74.0) years, $p=0.007)$. The age-related distributions in patients with GI involvement and renal involvement are presented in Fig. 1.

We measured the serum concentration of IgA in all patients. The pre-treatment IgA serum concentration exceeded the upper limit of reference in $102(47.7 \%)$ cases. The median (IQR) concentration in patients with an elevated serum IgA level was 5.26 (4.60-6.70) g/l (normal range $0.61-3.56 \mathrm{~g} / \mathrm{l}$ ). No patient had a subnormal serum IgA concentration. IgAV patients with renal involvement had significantly higher serum IgA concentrations compared to the subgroup with a GI involvement (median (IQR) of 4.4 (3.2-5.6) vs. $3.6(2.4-4.6) \mathrm{g} / \mathrm{l}, p=0.008)$.

Median (IQR) pre-treatment NLR in our IgAV cohort was 4.0 (2.6-5.9), range $0.8-55.0$. The ROC curve analysis demonstrated NLR $>3.5$ as the best cut-off value for the prediction of concurrent GI or renal involvement with $68.7 \%$ sensitivity, $52.5 \%$ specificity, and the area under the curve of 0.606 (95\%CI $0.537-0.672)$.

\section{IgAV treatment}

We treated the patients in line with the local practice. Indications for systemic immunosuppressive treatment were necrotic purpura, any GI involvement, or severe kidney involvement. IgAV spontaneously remitted in 48 (22.4\%) cases. Topical steroids were the only treatment in $21(9.8 \%)$ patients. We treated $61(28.5 \%)$ patients with intravenous methylprednisolone pulses (MP; 125$1000 \mathrm{mg} \mathrm{qd}$, for three consecutive days). In 17 patients, MP pulses were the only treatment, while the remaining 44 patients continued with oral glucocorticoids. We treated a total of $129(60.3 \%)$ patients with oral MP in a

Table 2 Characteristics of IgAV patients with and without gastrointestinal or renal involvement

\begin{tabular}{|c|c|c|c|c|c|c|}
\hline \multirow[t]{2}{*}{ Characteristics } & \multicolumn{3}{|c|}{ Gastrointestinal involvement } & \multicolumn{3}{|c|}{ Renal involvement } \\
\hline & Yes & No & $p$ value & Yes & No & $p$ value \\
\hline Number & 58 & 156 & & 83 & 131 & \\
\hline Disease duration (days) ${ }^{\#}$ & $8(7-15)$ & $7(5-21)$ & 0.650 & $7(5-19)$ & $7(5-21)$ & 0.682 \\
\hline Age (years) $^{\#}$ & $55.5(39.0-74.0)$ & $65.4(48.1-76.9)$ & 0.053 & $67.8(53.6-77.4)$ & $59.5(40.2-75.8)$ & 0.006 \\
\hline Male gender* & 70.7 & 55.1 & 0.043 & 61.4 & 58.0 & 0.669 \\
\hline Active smoking* & 22.4 & 18.6 & 0.563 & 26.5 & 15.3 & 0.052 \\
\hline Prior infection* & 34.5 & 31.4 & 0.743 & 32.5 & 32.1 & 1.0 \\
\hline Concurrent infection* & 10.3 & 17.3 & 0.287 & 16.9 & 14.5 & 0.699 \\
\hline New medication* & 25.9 & 23.7 & 0.724 & 24.1 & 24.4 & 1.0 \\
\hline Past cancer* & 6.9 & 14.7 & 0.165 & 12.0 & 13.0 & 1.0 \\
\hline Generalized purpura* & 79.3 & 40.4 & $<0.001$ & 60.2 & 45.0 & 0.036 \\
\hline Necrotic purpura* & 46.6 & 45.5 & 1.0 & 48.2 & 44.3 & 0.673 \\
\hline Elevated serum IgA* & 34.5 & 52.6 & 0.021 & 60.2 & 39.7 & 0.005 \\
\hline$N L R^{\#}$ & $4.89(3.3-6.7)$ & $3.8(2.4-5.5)$ & 0.003 & $4.6(2.9-6.5)$ & $3.8(2.4-5.5)$ & 0.041 \\
\hline
\end{tabular}




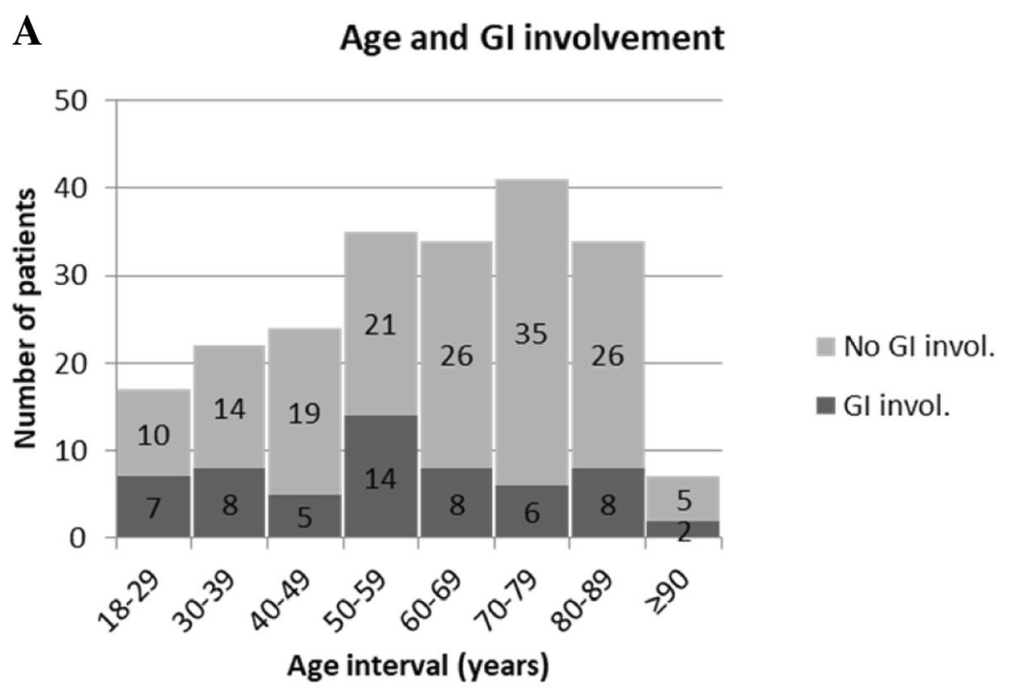

B Age and renal involvement

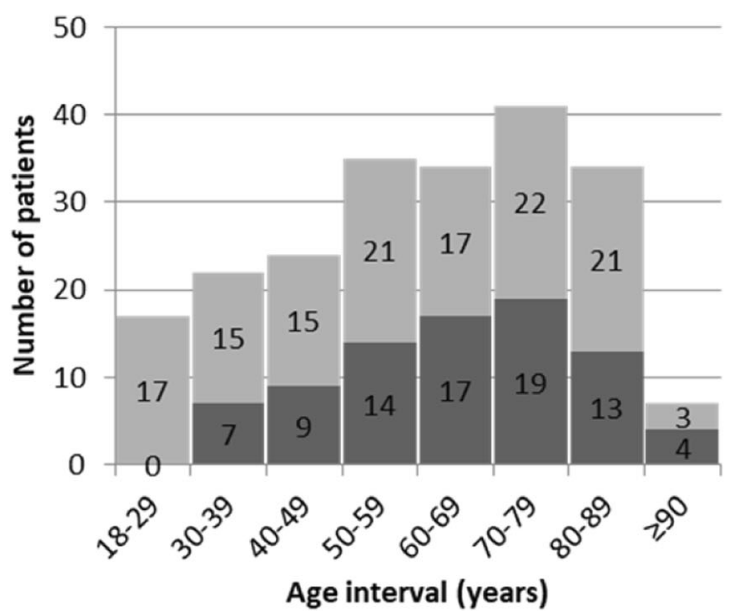

No renal invol.

Renal invol.

median (IQR) initial dose of 0.5 (0.4-0.7) $\mathrm{kg} /$ body weight. Thirty (14.0\%) patients were additionally treated with immunomodulatory therapy: cyclophosphamide (22, 10.3\%), intravenous immunoglobulins (9, 4.2\%), mycophenolate mofetil $(1,0.5 \%)$, rituximab (1, 0.5\%), dapsone $(2,0.9 \%)$, and colchicine $(2,0.9 \%)$. Three $(1.4 \%)$ patients had a plasma exchange, and $5(2.5 \%)$ patients additionally received montelukast. Three patients (1.4\%) with ischemic bowel perforation needed surgical intervention. Another two patients (0.9\%) underwent explorative laparoscopy. None of the patients required hemodialysis. Three (1.4\%) patients died during the acute phase of the disease: two died due to severe GI involvement and one due to pneumocystis pneumonia.

In the multivariate logistic regression analysis, severe IgAV (severe GI or severe renal involvement) (OR 8.86 (95\%CI 3.24-24.27), $p<0.001)$, renal involvement of any type (4.53 (95\%CI 1.51-13.54), $p=0.007$ ), generalized purpura (OR 3.30 (95\%CI 1.1-10.35), $p=0.040$ ), and female gender (OR 0.23 (95\%CI 0.07-0.76), $p=0.017$ ) were extracted as factors associated with the use of immunomodulatory therapy besides glucocorticoids.

\section{Predicting GI or renal involvement in IgAV}

In the multivariate logistic regression analysis adjusted for concurrent infection, generalized purpura (OR 6.74 $(95 \% \mathrm{CI}$ 3.18-14.31), $p<0.001$ ), pre-treatment NLR $>3.5$ (OR 2.78 (95\%CI 1.34-5.75), $p=0.006$ ), and elevated serum IgA level (OR $0.40(95 \%$ CI $0.20-0.79), p=0.009)$ were extracted as factors associated with any GI involvement (Table 3). In a subanalysis focused on 15 cases with severe GI involvement generalized purpura (OR 6.79 (95\%CI 1.38-33.53), $p=$ 0.019 ), NLR $>3.5$ (OR 11.28 (95\%CI 2.06-61.76), $p=$ $0.005)$, history of antecedent infection (OR 3.74 (95\%CI 
$1.09-12.85), p=0.036$ ), and age (OR 0.94 (95\%CI $0.91-$ $0.98), p=0.001$ ) emerged as factors associated with severe GI complications.

Active smoking (OR 3.23 (95\%CI 1.50-6.98), $p=$ 0.003), generalized purpura (OR 1.98 (95\%CI 1.08-3.61), $p=0.027$ ), elevated serum IgA (OR 2.25 (95\%CI 1.21$4.18), p=0.010)$, NLR > 3.5 (OR 1.96 (95\%CI 1.02-3.77), $p=0.044)$, and increasing patient age (1.02 (95\%CI $1.01-$ $1.04), p=0.012$ ) were extracted as factors associated with any renal involvement (Table 3 ). Active smoking (OR 4.42 (95\%CI 1.69-11.58), $p=0.002$ ), generalized purpura (OR 2.41 (95\%CI 1.03-5.61), $p=0.041$ ), and increasing age (OR 1.04 (95\%CI 1.01-1.07), $p=0.007$ ), but not serum IgA, emerged also as factors associated with acute kidney injury or nephrotic syndrome.

The composite outcome of either GI or renal involvement was associated with generalized purpura (OR 3.80 (95\%CI 2.10-6.88), $p<0.001$ ), active smoking (OR 2.51 (95\%CI 1.16-5.45), $p=0.020$ ), and NLR > 3.5 (OR 3.02 (95\%CI 1.63-5.60), $p<0.001$ ) (Table 3).

Disease duration prior to the diagnosis, patient gender, history of prior infection, use of new medication, history of cancer, and necrotic skin purpura at presentation were not significantly associated with GI or renal involvement in adult IgAV.

\section{Discussion}

In the present study, we evaluated predictors of GI and renal involvement, the two most commonly affected visceral organs in IgAV [10], in a prospectively collected cohort of adult IgAV patients. Although IgAV is usually considered a childhood disease with GI involvement reported in 50-75\%, and renal involvement in $40-50 \%$ of cases [11], the disease is not uncommon in adults [12]. In addition, the disease is frequently severe in adults, compared to commonly uneventful disease course in children [2].

In the majority of cases, GI and kidney inflammation followed the development of skin lesions. Particularly, renal involvement can be delayed for several weeks and can be missed [13] and can progress to chronic kidney disease $[2,3]$. In addition, severe GI involvement with bowel ischemia that leads to tissue necrosis and perforation or major bleeding represents the major source of morbidity and mortality in the acute phase of IgAV [14]. The risk for this devastating complication is especially increased in the elderly and the polymorbid patients. Therefore, the identification of markers predicting the two most frequent visceral manifestations of IgAV would considerably ease the routine clinical work.

Our study shows that a generalized purpura (i.e., purpura above the waistline), and not skin necroses, predicts both renal and GI involvement in adult IgAV. As previously shown, generalized purpura in smokers also predicted the severity of kidney and GI involvement [15]. Our present findings are in contrast with those of Cao et al., and Poterucha et al., who found a correlation between necrotic purpura and visceral involvement, and the absence of a correlation with the extent of skin lesions and visceral involvement, respectively $[4,5]$. Nevertheless, similarly to Poterucha et al., we noticed a trend toward opposing age-related distribution between patients with GI and those with renal involvementadult patients with GI involvement being significantly younger than those with renal involvement [5].

Active smoking emerged as a potential risk factor for renal involvement in adult IgAV. The association between smoking and the progression of chronic kidney disease has been appreciated in diabetic and several non-diabetic chronic kidney diseases, including IgA nephropathy $[16$, 17]. Cha et al. demonstrated a link between the progression of IgA nephropathy and smoking-related injury of the microvasculature [16]. In animal and in vitro models, nicotine activated inflammatory mediators, induced mesangial cell proliferation, and fibronectin production [18].

In our study, the serum IgA concentrations were divergent in patients with GI and renal involvement. Patients with the elevated serum IgA seem to have a lower risk of GI tract involvement but a higher risk of renal involvement. A lower frequency of elevated serum IgA in GI involvement has not been previously reported. Data in the literature is scarce; nevertheless, a recent study in children found significantly lower serum IgA in IgAV patients with scrotal involvement versus those without this manifestation [19]. And a study on biomarkers of childhood IgAV nephritis showed no significant differences in the serum IgA level comparing cases with and without nephritis [20].

Table 3 Predictors of gastrointestinal and renal involvement using multiple logistic regression

\begin{tabular}{|c|c|c|c|c|c|c|}
\hline \multirow[t]{2}{*}{ Characteristics } & \multicolumn{2}{|l|}{ Gl involvement } & \multicolumn{2}{|c|}{ Renal involvement } & \multicolumn{2}{|c|}{ Gl or renal involvement } \\
\hline & OR $(95 \% \mathrm{Cl})$ & $p$ value & OR $(95 \% \mathrm{Cl})$ & $p$ value & OR $(95 \% \mathrm{Cl})$ & $p$ value \\
\hline Age & - & - & $1.02(1.01-1.04)$ & 0.023 & - & - \\
\hline Active smoking & - & - & $3.23(1.50-6.98)$ & 0.003 & $2.51(1.16-5.45)$ & 0.020 \\
\hline Generalized purpura & $6.74(3.18-4.31)$ & $<0.001$ & $2.00(1.08-3.61)$ & 0.027 & $3.80(2.10-6.88)$ & $<0.001$ \\
\hline Elevated serum IgA & $0.40(0.20-0.79)$ & 0.009 & $2.25(1.21-4.18)$ & 0.010 & - & - \\
\hline$N L R>3.5$ & $2.78(1.34-5.75)$ & 0.006 & $1.96(1.02-3.77)$ & 0.044 & $3.02(1.63-5.60)$ & $<0.001$ \\
\hline
\end{tabular}


The predictive roles of elevated pre-treatment NLR as a marker of systemic involvement in IgAV and a marker reflecting IgAV inflammatory response have been reported. Nagy et al. found that pre-treatment NLR $>3.3$ correlated with the severity of the disease in patients who developed GI and renal involvement [7]. Similarly, we also found that higher NLR was associated with GI and renal involvement. The optimal cut-off in our cohort was NLR $>3.5$. However, as it had poor sensitivity and specificity, it should not be interpreted in isolation.

Our study had several strengths. We prospectively investigated 214 adult patients with IgAV proven by skin biopsy and elucidated risk factors of severe involvement. However, the present study also had limitations: (1) cumulative smoking exposure, (2) a factor of passive smoking, and (3) long-term IgAV prognosis have not been analyzed due to lack of information. Because of the small number of patients who underwent renal biopsy, the histological features of kidney involvement could not be analyzed.

\section{Conclusion}

Gastrointestinal or renal involvement in adult IgAV could be predicted by smoking status, distribution of skin lesions, and the neutrophil to lymphocyte ratio. The presented findings could help physicians identify the patients at risk who need vigilant monitoring during the acute phase of the disease.

\section{Abbreviations \\ Cl: Confidence interval; EULAR/PRINTO/PRES: European League Against Rheumatism/Paediatric Rheumatology International Trials Organization/ Paediatric Rheumatology European Society; Gl: Gastrointestinal; IgAV: Immunoglobulin A vasculitis; IQR: Interquartile range; MP: Methylprednisolone; NLR: Neutrophil to lymphocyte ratio; OR: Odds ratio; ROC: Receiver operating characteristic; SD: Standard deviation; WBC: White blood cell count}

\section{Acknowledgements}

Not applicable.

\section{Authors' contributions}

All authors meet the authorship requirements. AH contributed to the acquisition, analysis, and interpretation of data. VJ contributed to the interpretation of histological analyses. MT, KPP, and ŽR helped to revise the manuscript and gave suggestions. All authors have read and approved the final manuscript for submission.

\section{Funding}

The study was funded by the Slovenian Research Agency-research core funding P3-0314.

\section{Availability of data and materials}

The datasets analyzed in the current study are available from the corresponding author on request.

\section{Ethics approval and consent to participate}

The study was approved by the Slovenian National medical ethics committee, approval number 159/07/13.

All patients included in the study provided informed consent to participate.

\section{Consent for publication}

Not applicable.

\section{Competing interests}

The authors declare that they have no competing interests.

\section{Author details}

'Department of Rheumatology, University Medical Centre Ljubljana, Vodnikova cesta 62, 1000 Ljubljana, Slovenia. ${ }^{2}$ Faculty of Medicine, University of Ljubljana, Ljubljana, Slovenia. ${ }^{3}$ Faculty of Medicine, Institute of Pathology, University of Ljubljana, Ljubljana, Slovenia.

Received: 20 October 2019 Accepted: 16 December 2019

Published online: 26 December 2019

\section{References}

1. Jennette JC, Falk RJ, Bacon PA, et al. 2012 revised International Chapel Hill Consensus Conference Nomenclature of Vasculitides. Arthritis Rheum. 2013; 65:1-11. https://doi.org/10.1002/art.37715.

2. Pillebout E, Thervet E, Hill G, Alberti C, Vanhille P, Nochy D. Henoch-Schonlein Purpura in adults: outcome and prognostic factors. J Am Soc Nephrol. 2002;13: 1271-8. https://doi.org/10.1097/01.asn.0000013883.99976.22.

3. Coppo R, Andrulli S, Amore A, Gianoglio B, Conti G, Peruzzi L, et al. Predictors of outcome in Henoch-Schonlein nephritis in children and adults. Am J Kidney Dis. 2006;47:993-1003. https://doi.org/10.1053/j.ajkd.2006.02.178.

4. Cao R, Lau S, Tan V, Tey HL. Adult Henoch-Schonlein purpura: clinical and histopathological predictors of systemic disease and profound renal disease. Indian J Dermatol Venereol Leprol. 2017. https://doi.org/10.4103/ijdvl. IJDVL_571_16.

5. Poterucha TJ, Wetter DA, Gibson LE, Camilleri MJ, Lohse CM. Correlates of systemic disease in adult Henoch-Schönlein purpura: a retrospective study of direct immunofluorescence and skin lesion distribution in 87 patients at Mayo Clinic. J Am Acad Dermatol. 2012 Oct;67(4):612-6. https://doi.org/10.1016/.jaad.2011.11.946.

6. St John J, Vedak P, Garza-Mayers AC, Hoang MP, Nigwekar SU, Kroshinsky D. Location of skin lesions in Henoch-Schönlein purpura and its association with significant renal involvement. J Am Acad Dermatol. 2018 Jan;78(1):11520. https://doi.org/10.1016/j.jaad.2017.04.1122.

7. Nagy GR, Kemény L, Bata-Csörgő Z. Neutrophil-to-lymphocyte ratio: a biomarker for predicting systemic involvement in adult IgA vasculitis patients. J Eur Acad Dermatol Venereol. 2017 Jun;31(6):1033-7. https://doi. org/10.1111/jdv.14176.

8. Ekinci RMK, Balci S, Sari Gokay S, Yilmaz HL, Dogruel D, Altintas DU, et al. Do practical laboratory indices predict the outcomes of children with HenochSchönlein purpura? Postgrad Med. 2019 May;131(4):295-8. https://doi.org/ 10.1080/00325481.2019.1609814

9. Ozen S, Pistorio A, lusan SM, Bakkaloglu A, Herlin T, Brik R, et al. EULAR/ PRINTO/PRES criteria for Henoch-Schönlein purpura, childhood polyarteritis nodosa, childhood Wegener granulomatosis and childhood Takayasu arteritis: Ankara 2008. Part II: final classification criteria. Ann Rheum Dis. 2010 May;69(5):798-806. https://doi.org/10.1136/ard.2009.116657.

10. Hocevar A, Rotar Z, Jurcic V, Cucnik S, Tomšič M. Patient age, gender and extent of purpura may suggest short-term outcomes in adults with IgA vasculitis. Rheumatology (Oxford). 2015;54:1330-2. https://doi.org/10.1093/ rheumatology/kev122.

11. Saulsbury FT. Henoch-Schönlein purpura. Curr Opin Rheumatol. 2010 Sep; 22(5):598-602. https://doi.org/10.1097/BOR.0b013e32833af608.

12. Hočevar A, Rotar Z, Ostrovršnik J, Jurčić V, Vizjak A, Dolenc Voljč M, et al. Incidence of IgA vasculitis in the adult Slovenian population. $\mathrm{Br} J$ Dermatol. 2014 Sep;171(3):524-7. https://doi.org/10.1111/bjd.12946.

13. Narchi H. Risk of long term renal impairment and duration of follow up recommended for Henoch-Schonlein purpura with normal or minimal urinary findings: a systematic review. Arch Dis Child. 2005;90:916-20. https:/ doi.org/10.1136/adc.2005.074641.

14. Ebert EC. Gastrointestinal manifestations of Henoch-Schonlein Purpura. Dig Dis Sci. 2008 Aug;53(8):2011-9. https://doi.org/10.1007/s10620-007-0147-0.

15. Hocevar A, Rotar Z, Kejzar N, Tomsic M. A model predicting short term severity of IgA vasculitis in adults. J Clin Exp Dermatol Res. 2017;8:397. https://doi.org/10.4172/2155-9554.1000397.

16. Cha YJ, Lim BJ, Kim BS, Kim Y, Yoo TH, Han SH, et al. Smoking-related renal histologic injury in IgA nephropathy patients. Yonsei Med J. 2016;57:209-16. https://doi.org/10.3349/ymj.2016.57.1.209.

17. Yamamoto R, Nagasawa Y, Shoji T, Iwatani H, Hamano T, Kawada N, et al. Cigarette smoking and progression of IgA nephropathy. Am J Kidney Dis. 2010;56:313-24. https://doi.org/10.1053/j.ajkd.2010.02.351. 
18. Jaimes EA, Tian RX, Joshi MS, Raij L. Nicotine augments glomerular injury in a rat model of acute nephritis. Am J Nephrol. 2009;29:319-26. https://doi. org/10.1159/000163593.

19. Buscatti IM, Abrão HM, Kozu K, VLS M, Gomes RC, AME S, et al.

Characterization of scrotal involvement in children and adolescents with IgA vasculitis. dv Rheumatol. 2018;58(1):38. https://doi.org/10.1186/s42358018-0039-3.

20. Pillebout $E$, Jamin A, Ayari $H$, Housset $P$, Pierre $M$, Sauvaget $V$, et al. Biomarkers of IgA vasculitis nephritis in children. PLoS One. 2017;12(11): e0188718. https://doi.org/10.1371/journal.pone.0188718.

\section{Publisher's Note}

Springer Nature remains neutral with regard to jurisdictional claims in published maps and institutional affiliations.

Ready to submit your research? Choose BMC and benefit from:

- fast, convenient online submission

- thorough peer review by experienced researchers in your field

- rapid publication on acceptance

- support for research data, including large and complex data types

- gold Open Access which fosters wider collaboration and increased citations

- maximum visibility for your research: over $100 \mathrm{M}$ website views per year

At BMC, research is always in progress.

Learn more biomedcentral.com/submissions 